\title{
Short communication: Alteration of priors for random effects in Gaussian linear mixed models
}

\author{
J. Vandenplas,${ }^{*} \dagger^{1}$ O. F. Christensen, $\ddagger$ and N. Gengler* \\ *Animal Science Unit, Gembloux Agro Bio-Tech, University of Liege, 5030 Gembloux, Belgium \\ †National Fund for Scientific Research, 1000 Brussels, Belgium \\ $\ddagger$ Center for Quantitative Genetics and Genomics, Dept. Molecular Biology and Genetics, Aarhus University, 8830 Tjele, Denmark
}

\section{ABSTRACT}

Linear mixed models, for which the prior multivariate normal distributions of random effects are assumed to have a mean equal to 0 , are commonly used in animal breeding. However, some statistical analyses (e.g., the consideration of a population under selection into a genomic scheme breeding, multiple-trait predictions of lactation yields, and Bayesian approaches integrating external information into genetic evaluations) need to alter both the mean and (co)variance of the prior distributions and, to our knowledge, most software packages available in the animal breeding community do not permit such alterations. Therefore, the aim of this study was to propose a method to alter both the mean and (co)variance of the prior multivariate normal distributions of random effects of linear mixed models while using currently available software packages. The proposed method was tested on simulated examples with 3 different software packages available in animal breeding. The examples showed the possibility of the proposed method to alter both the mean and (co)variance of the prior distributions with currently available software packages through the use of an extended data file and a user-supplied (co)variance matrix.

Key words: prior distribution, Bayesian, linear mixed model

\section{Short Communication}

Currently, Henderson's mixed models methods and BLUP (Henderson, 1975) are commonly used in animal breeding. The typical linear mixed model is written as follows:

$$
\mathbf{y}=\mathbf{X} \boldsymbol{\beta}+\mathbf{Z u}+\mathbf{e}
$$

where $\mathbf{y}$ is the vector of records; $\boldsymbol{\beta}$ and $\mathbf{u}$ are vectors of fixed and random effects related to the records through

Received March 21, 2014

Accepted June 9, 2014.

${ }^{1}$ Corresponding author: jvandenplas@ulg.ac.be the incidence matrices $\mathbf{X}$ and $\mathbf{Z}$, respectively; and $\mathbf{e}$ is the vector of residuals.

It is assumed that the expectations are

$$
E\left[\begin{array}{l}
\mathbf{u} \\
\mathbf{e}
\end{array}\right]=\left[\begin{array}{l}
\mathbf{0} \\
\mathbf{0}
\end{array}\right]
$$

and the (co)variance matrices are

$$
\operatorname{Var}\left[\begin{array}{l}
\mathbf{u} \\
\mathbf{e}
\end{array}\right]=\left[\begin{array}{cc}
\mathbf{G} & \mathbf{0} \\
\mathbf{0} & \mathbf{R}
\end{array}\right]
$$

where $\mathbf{G}$ is the (co)variance matrix associated with $\mathbf{u}$ and $\mathbf{R}$ is the (co) variance matrix associated with $\mathbf{e}$. The estimates of $\boldsymbol{\beta}$; that is, $\hat{\boldsymbol{\beta}}$, and the predictions of $\mathbf{u}$; that is, $\hat{\mathbf{u}}$, can be obtained solving the mixed-model equations written as follows (Henderson, 1950):

$$
\left[\begin{array}{cc}
\mathbf{X}^{\prime} \mathbf{R}^{-1} \mathbf{X} & \mathbf{X}^{\prime} \mathbf{R}^{-1} \mathbf{Z} \\
\mathbf{Z}^{\prime} \mathbf{R}^{-1} \mathbf{X} & \mathbf{Z}^{\prime} \mathbf{R}^{-1} \mathbf{Z}+\mathbf{G}^{-1}
\end{array}\right]\left[\begin{array}{l}
\hat{\boldsymbol{\beta}} \\
\hat{\mathbf{u}}
\end{array}\right]=\left[\begin{array}{l}
\mathbf{X}^{\prime} \mathbf{R}^{-1} \mathbf{y} \\
\mathbf{Z}^{\prime} \mathbf{R}^{-1} \mathbf{y}
\end{array}\right] .
$$

In the case of BLUP, the (co)variance matrices $\mathbf{G}$ and $\mathbf{R}$ are assumed to be known.

Although $\mathbf{u}$ is assumed to have an expectation equal to $\mathbf{0}$, a need exists to alter this expectation in some statistical analyses. For example, Bayesian approaches integrating external information (i.e., EBV and associated reliabilities obtained from a foreign genetic evaluation) into a local genetic evaluation alter both $E[\mathbf{u}]$ and $\operatorname{Var}[\mathbf{u}]$ (e.g., Gianola and Fernando, 1986; Quaas and Zhang, 2006; Legarra et al., 2007). For such approaches, $E[\mathbf{u}]$ is equal to the foreign EBV and $\operatorname{Var}[\mathbf{u}]$ represents the associated matrix of prediction error (co)variances. Another example is the consideration of a population under selection into a genomic scheme breeding by assuming $E[\mathbf{u}] \neq \mathbf{0}$ for the genotyped animals. Indeed, they may have an expectation different from 0 if selection occurred (Vitezica et al., 2011). Also, Schaeffer and Jamrozik (1996) proposed a multiple-trait procedure for predicting lactation yields for dairy cows based on an alteration of $E[\mathbf{u}]$ with information from groups 
of cows sharing the same production characteristics. However, to our knowledge, most software packages currently available in animal breeding do not permit alterations of expectations of random effects, whereas they may allow the use of a user supplied covariance matrix. Therefore, the aim of this study was to propose a method to alter both the expectations and (co)variances of random effects while using software packages currently available in animal breeding. The development of the proposed method was based on a Bayesian view of linear mixed models.

\section{Bayesian View of Linear Mixed Models}

Bayes estimators for linear (mixed) models and their relations with BLUP were discussed by several authors (e.g., Lindley and Smith, 1972; Dempfle, 1977; Gianola and Fernando, 1986; Sorensen and Gianola, 2002). From a Bayesian view, all fixed and random effects are considered as random. However, the terms "fixed" and "random" will still be used below to differentiate $\boldsymbol{\beta}$ from u, respectively.

For the linear model 1, the following prior multivariate normal $(M V N)$ distributions are assumed:

$$
[\boldsymbol{\beta} \mid \mathbf{B}] \sim \operatorname{MVN}(\mathbf{b}, \mathbf{B}),
$$

where $\mathbf{b}$ is a mean vector and $\mathbf{B}$ is a (co)variance matrix,

$$
[\mathbf{u} \mid \mathbf{G}] \sim \operatorname{MVN}(\mathbf{g}, \mathbf{G}),
$$

where $\mathbf{g}$ is a mean vector, and

$$
[\mathbf{e} \mid \mathbf{R}] \sim \operatorname{MVN}(\mathbf{0}, \mathbf{R}) .
$$

Assuming that all the (co)variance matrices are known, the joint posterior density of $\boldsymbol{\beta}$ and $\mathbf{u}$ can be written as follows:

$$
\begin{aligned}
& f(\boldsymbol{\beta}, \mathbf{u} \mid \mathbf{y}, \mathbf{B}, \mathbf{G}, \mathbf{R}) \\
& \propto \exp \left\{-\frac{1}{2}\left[\begin{array}{l}
(\mathbf{y}-\mathbf{X} \boldsymbol{\beta}-\mathbf{Z u})^{\prime} \mathbf{R}^{-1}(\mathbf{y}-\mathbf{X} \boldsymbol{\beta}-\mathbf{Z u}) \\
+(\boldsymbol{\beta}-\mathbf{b})^{\prime} \mathbf{B}^{-1}(\boldsymbol{\beta}-\mathbf{b})+(\mathbf{u}-\mathbf{g})^{\prime} \mathbf{G}^{-1}(\mathbf{u}-\mathbf{g})
\end{array}\right]\right\} .
\end{aligned}
$$

Because this joint posterior distribution is multivariate normal, its mean equals its mode, and $\boldsymbol{\beta}$ and $\mathbf{u}$ can be estimated by differentiating the joint posterior distribution with respect to $\boldsymbol{\beta}$ and $\mathbf{u}$ and setting the derivatives equal to zero. From this, as shown by Gianola and Fernando (1986), the following equation is obtained:

$$
\left[\begin{array}{cc}
\mathbf{X}^{\prime} \mathbf{R}^{-1} \mathbf{X}+\mathbf{B}^{-1} & \mathbf{X}^{\prime} \mathbf{R}^{-1} \mathbf{Z} \\
\mathbf{Z}^{\prime} \mathbf{R}^{-1} \mathbf{X} & \mathbf{Z}^{\prime} \mathbf{R}^{-1} \mathbf{Z}+\mathbf{G}^{-1}
\end{array}\right]\left[\begin{array}{c}
\hat{\boldsymbol{\beta}} \\
\hat{\mathbf{u}}
\end{array}\right]=\left[\begin{array}{c}
\mathbf{X}^{\prime} \mathbf{R}^{-1} \mathbf{y}+\mathbf{B}^{-1} \mathbf{b} \\
\mathbf{Z}^{\prime} \mathbf{R}^{-1} \mathbf{y}+\mathbf{G}^{-1} \mathbf{g}
\end{array}\right] .
$$

If a noninformative prior is considered for $\boldsymbol{\beta}$ (i.e., $\left.\mathbf{B}^{-1} \rightarrow \mathbf{0}\right)$ and $\mathbf{g}=\mathbf{0}$, the system of equations [3] simplifies to traditional mixed-model equations [2].

\section{Alteration of Priors for Random Effects}

In the following development, it is assumed that $\mathrm{g}$ and $\mathbf{G}^{-1}$ are known, $\mathbf{g} \neq \mathbf{0}$, and a noninformative prior for $\boldsymbol{\beta}$ is considered. Therefore, the system of equations $[3]$ is written as follows:

$$
\left[\begin{array}{cc}
\mathbf{X}^{\prime} \mathbf{R}^{-1} \mathbf{X} & \mathbf{X}^{\prime} \mathbf{R}^{-1} \mathbf{Z} \\
\mathbf{Z}^{\prime} \mathbf{R}^{-1} \mathbf{X} & \mathbf{Z}^{\prime} \mathbf{R}^{-1} \mathbf{Z}+\mathbf{G}^{-1}
\end{array}\right]\left[\begin{array}{c}
\hat{\boldsymbol{\beta}} \\
\hat{\mathbf{u}}
\end{array}\right]=\left[\begin{array}{c}
\mathbf{X}^{\prime} \mathbf{R}^{-1} \mathbf{y} \\
\mathbf{Z}^{\prime} \mathbf{R}^{-1} \mathbf{y}+\mathbf{G}^{-1} \mathbf{g}
\end{array}\right] .
$$

Although most available software packages allow the use of a user-supplied (co)variance matrix as $\mathbf{G}$, or its inverse $\mathbf{G}^{-1}$, most of them do not allow that $\mathbf{g} \neq \mathbf{0}$. Thereby, the system of equations [4], allowing an alteration of the default mean, cannot be solved with current software packages. A way to solve this issue is to develop a system of equations equivalent to the system of equations [4] that can be solved by current software packages. Therefore, below we define $\mathbf{y}_{\mathbf{P}}$, a vector of pseudo-records (i.e., records corrected for all other effects than $\mathbf{u}$ ); $\mathbf{X}_{\mathbf{P}}$ and $\mathbf{Z}_{\mathbf{P}}, 2$ incidence matrices relating pseudo-records to $\boldsymbol{\beta}$ and $\mathbf{u}$, respectively; $\mathbf{R}_{\mathbf{P}}$, a residual (co)variance matrix associated to the pseudorecords; and $\mathbf{G}^{*}$, a (co)variance matrix associated with $\mathbf{u}$ conditional on pseudo-records. Assuming that $\mathbf{X}_{\mathbf{P}}=$ $\mathbf{0}, \mathbf{Z}_{\mathbf{P}}^{\prime} \mathbf{R}_{\mathbf{P}}^{-1} \mathbf{y}_{\mathbf{P}}=\mathbf{G}^{-1} \mathbf{g}$, and $\mathbf{G}^{-1}=\mathbf{G}^{*^{-1}}+\mathbf{Z}_{\mathbf{P}}^{\prime} \mathbf{R}_{\mathbf{P}}^{-1} \mathbf{Z}_{\mathbf{P}}$, the system of equations [4] is equivalent to

$$
\begin{aligned}
& {\left[\begin{array}{cc}
\mathbf{X}^{\prime} \mathbf{R}^{-1} \mathbf{X}+\mathbf{X}_{\mathbf{P}}^{\prime} \mathbf{R}_{\mathbf{P}}^{-1} \mathbf{X}_{\mathbf{P}} & \mathbf{X}^{\prime} \mathbf{R}^{-1} \mathbf{Z}+\mathbf{X}_{\mathbf{P}}^{\prime} \mathbf{R}_{\mathbf{P}}^{-1} \mathbf{Z}_{\mathbf{P}} \\
\mathbf{Z}^{\prime} \mathbf{R}^{-1} \mathbf{X}+\mathbf{Z}_{\mathbf{P}}^{\prime} \mathbf{R}_{\mathbf{P}}^{-1} \mathbf{X}_{\mathbf{P}} & \mathbf{Z}^{\prime} \mathbf{R}^{-1} \mathbf{Z}+\mathbf{G}^{*-1}+\mathbf{Z}_{\mathbf{P}}^{\prime} \mathbf{R}_{\mathbf{P}}^{-1} \mathbf{Z}_{\mathbf{P}}
\end{array}\right]\left[\begin{array}{c}
\hat{\boldsymbol{\beta}} \\
\hat{\mathbf{u}}
\end{array}\right]} \\
& =\left[\begin{array}{c}
\mathbf{X}^{\prime} \mathbf{R}^{-1} \mathbf{y}+\mathbf{X}_{\mathbf{P}}^{\prime} \mathbf{R}_{\mathbf{P}}^{-1} \mathbf{y}_{\mathbf{P}} \\
\mathbf{Z}^{\prime} \mathbf{R}^{-1} \mathbf{y}+\mathbf{Z}_{\mathbf{P}}^{\prime} \mathbf{R}_{\mathbf{P}}^{-1} \mathbf{y}_{\mathbf{P}}
\end{array}\right] .
\end{aligned}
$$

The system of equations [5] can be written using compact notation as

$$
\left[\begin{array}{cc}
\mathbf{X}^{* \prime} \mathbf{R}^{*-1} \mathbf{X}^{*} & \mathbf{X}^{* \prime} \mathbf{R}^{*-1} \mathbf{Z}^{*} \\
\mathbf{Z}^{* \prime} \mathbf{R}^{*-1} \mathbf{X}^{*} & \mathbf{Z}^{* \prime} \mathbf{R}^{*-1} \mathbf{Z}^{*}+\mathbf{G}^{*-1}
\end{array}\right]\left[\begin{array}{l}
\hat{\boldsymbol{\beta}} \\
\hat{\mathbf{u}}
\end{array}\right]=\left[\begin{array}{l}
\mathbf{X}^{* \prime} \mathbf{R}^{*-1} \mathbf{y} * \\
\mathbf{Z}^{* \prime} \mathbf{R}^{*-1} \mathbf{y}^{*}
\end{array}\right]
$$


where

$$
\begin{aligned}
& \mathbf{X}^{*}=\left[\begin{array}{c}
\mathbf{X} \\
\mathbf{X}_{\mathbf{P}}
\end{array}\right], \mathbf{Z}^{*}=\left[\begin{array}{c}
\mathbf{Z} \\
\mathbf{Z}_{\mathbf{P}}
\end{array}\right], \mathbf{y}^{*}=\left[\begin{array}{c}
\mathbf{y} \\
\mathbf{y}_{\mathbf{P}}
\end{array}\right], \\
& \text { and } \mathbf{R}^{*-1}=\left[\begin{array}{cc}
\mathbf{R}^{-1} & \mathbf{0} \\
\mathbf{0} & \mathbf{R}_{\mathbf{P}}^{-1}
\end{array}\right]
\end{aligned}
$$

Because the equivalent system of equations [5] has the same form as the system of equations [2], it can be solved using current software packages allowing the use of a user-supplied (co)variance matrix as $\mathbf{G}^{*}$, or its inverse $\mathbf{G}^{*-1}$, and a data file containing actual records $(\mathbf{y})$ and extended with pseudo-records $\left(\mathbf{y}_{\mathbf{P}}\right)$. Therefore, $\mathbf{y}_{\mathbf{P}}$ and $\mathbf{G}^{*^{-1}}$ must be computed before solving the system of equations [5] as follows (after some computational simplifications):

(1) Set up the matrix $\mathbf{G}^{-1}$ and the vector $\mathbf{g}$;

(2) Compute $\boldsymbol{\theta}=\mathbf{G}^{-1} \mathbf{g}$;

(3) Set up the matrix $\mathbf{Z}_{\mathbf{P}}$, which is a diagonal matrix with (a) $\mathbf{Z}_{\mathbf{P}_{i j}}=1$ if $\boldsymbol{\theta}_{i} \neq 0$ or (b) $\mathbf{Z}_{\mathbf{P}_{i i}}=0$ if $\boldsymbol{\theta}_{i}=0$, where $i=1, \ldots, n$ and $n$ is the number of levels associated with $\mathbf{u}$;

(4) Compute the (co)variance matrix $\mathbf{R}_{\mathbf{P}}$ as $\mathbf{R}_{\mathbf{P}}=$ $\mathbf{Z}_{\mathbf{P}}\left(\mathbf{I} \otimes \mathbf{R}_{0}\right) \mathbf{Z}_{\mathbf{P}}$, where $\mathbf{I}$ is an identity matrix of size $k$ equal to the number of records for each trait (for simplicity assumed to be the same across traits) and $\mathbf{R}_{0}$ is the residual (co)variance matrix between traits for 1 record;

(5) Compute the vector of pseudo-records $\mathbf{y}_{\mathbf{P}}$ as $\mathbf{y}_{\mathbf{P}}$ $=\mathbf{R}_{\mathbf{P}} \boldsymbol{\theta}$

(6) Compute the (co)variance matrix $\mathbf{G}^{*^{-1}}$ as $\mathbf{G}^{*-1}=\mathbf{G}^{-1}-\mathbf{R}_{\mathbf{P}}^{-1}$, where $\mathbf{R}_{\mathbf{P}}^{-1}$ is the generalized inverse of $\mathbf{R}_{\mathbf{P}}$.

Each pseudo-record in $\mathbf{y}_{\mathbf{P}}$ must be added to the data file. Because each pseudo-record can be considered as 1 record corrected for all effects other than $\mathbf{u}$, all fixed effects are set to 0 , leading to $\mathbf{X}_{\mathbf{P}}=\mathbf{0}$, except for the effect $\mathbf{u}$, for which it is equal to the level associated with the pseudo-record. With this approach, the system of equations [5], equivalent to the system of equations [4], can be solved using current software packages.

For a univariate analysis, step (4) is not needed and the computations of steps (5) and (6) can be simplified to $\mathbf{y}_{\mathbf{P}}=\boldsymbol{\theta} \sigma_{e}^{2}$ and $\mathbf{G}^{*-1}=\mathbf{G}^{-1}-\mathbf{Z}_{\mathbf{P}}\left(\sigma_{e}^{2}\right)^{-1}$, respectively, where $\sigma_{e}^{2}$ is the residual variance. For a multivariate analysis, software packages may only allow the use of a user-supplied matrix A (e.g., a relationships matrix), or its inverse $\mathbf{A}^{-1}$, instead of $\mathbf{G}$ or $\mathbf{G}^{-1}$, such that $\mathbf{G}^{-1}$ is equal to $\mathbf{G}^{-1}=\mathbf{A}^{-1} \otimes \mathbf{G}_{0}^{-1}$, where $\mathbf{G}_{0}^{-1}$ is the inverse of a known (co)variance matrix between traits. Because no matrix $\mathbf{A}^{*}$ or $\mathbf{A}^{*-1}$ can be found such that the computed $\mathbf{G}^{*^{-1}}$ is equal to $\mathbf{G}^{*^{-1}}=\mathbf{A}^{*^{-1}} \otimes \mathbf{G}_{0}^{-1}$, a canonical transformation of the multivariate model must be performed (e.g., Quaas, 1984). Therefore, a vector of transformed observations $\mathbf{y}_{\mathbf{T}}$ is defined such that $\mathbf{y}_{\mathbf{T} i}=$ $\mathbf{T} \mathbf{y}_{i}$ for the $i$ th animal $(i=1,2, \ldots, k)$, with a matrix $\mathbf{T}$ satisfying $\mathbf{T} \mathbf{R}_{0} \mathbf{T}^{\prime}=\mathbf{I}$ and $\mathbf{T} \mathbf{G}_{0} \mathbf{T}^{\prime}=\mathbf{D}$, where $\mathbf{D}$ is a diagonal matrix, and in addition, a vector of transformed prior means $\mathbf{g}_{\mathbf{T}}$ must be computed as $\mathbf{g}_{\mathbf{T} i}=\mathbf{T g}_{i}$ for $i=1,2, \ldots, k$. For the $j$ th transformed variable, the system of equations [4] can be then written as

$$
\left[\begin{array}{cc}
\mathbf{X}_{j}^{\prime} \mathbf{X}_{j} & \mathbf{X}_{j}^{\prime} \mathbf{Z}_{j} \\
\mathbf{Z}_{j}^{\prime} \mathbf{X}_{j} & \mathbf{Z}_{j}^{\prime} \mathbf{Z}_{j}+\mathbf{G}_{\mathbf{T} j}^{-1}
\end{array}\right]\left[\begin{array}{l}
\hat{\boldsymbol{\beta}}_{\mathbf{T} j} \\
\hat{\mathbf{u}}_{\mathbf{T} j}
\end{array}\right]=\left[\begin{array}{c}
\mathbf{X}_{j}^{\prime} \mathbf{y}_{\mathbf{T} j} \\
\mathbf{Z}_{j}^{\prime} \mathbf{y}_{\mathbf{T} j}+\mathbf{G}_{\mathbf{T} j}^{-1} \mathbf{g}_{\mathbf{T} j}
\end{array}\right],
$$

where $\mathbf{G}_{\mathbf{T} j}^{-1}=\mathbf{A}^{-1} \mathbf{D}_{j j}^{-1}$ is the inverse of the (co)variance matrix associated with $\mathbf{u}_{\mathbf{T} j}$ and $\mathbf{D}_{j j}^{-1}$ is the inverse of the $j$ th diagonal element of $\mathbf{D}$.

Because each analysis for the jth transformed variable is a univariate analysis, the limitation of the use of a user supplied matrix $\mathbf{A}$ or $\mathbf{A}^{-1}$ for a multivariate analysis is avoided. Thereby, the system of equations [6] can be solved for each $j$ th transformed variable with the system of equations [5]. The vector $\mathbf{y}_{\mathbf{P}}$ and the matrix $\mathbf{G}^{*-1}$ specific to the $j$ th transformed variable (i.e., $\mathbf{y}_{\mathbf{P} j}$ and $\left.\mathbf{G}_{j}^{*-1}\right)$ can be computed as described previously, and a matrix $\mathbf{A}_{j}^{*-1}$ can be found such that $\mathbf{G}_{j}^{*-1}=\mathbf{A}_{j}^{*-1} \mathbf{D}_{j j}^{-1}$. Solutions for the system of equations [4] expressed on the original scale are equal to $\hat{\boldsymbol{\beta}}_{i}=\mathbf{T}^{-1} \hat{\boldsymbol{\beta}}_{\mathbf{T} i}$ and $\hat{\mathbf{u}}_{i}=\mathbf{T}^{-1} \hat{\mathbf{u}}_{\mathbf{T} i}$ for $i=1,2, \ldots, k$.

If a software package does not accept levels of effects defined as 0 for a (pseudo-)record (e.g., DMU; Madsen and Jensen, 2012), solutions of [5] can be obtained by adding a dummy level for those effects to replace the level defined as 0 and then by writing a regression model where effects including a dummy level are each considered as a regression without intercept nested within the effects. The associated covariables are equal to 0 for the dummy levels; otherwise, they are equal to 1.

\section{Example 1: Numerical Example}

Consider a sample of 6 animals as designed in Table 1. Milk yields $(\mathrm{kg})$ for animals 2 to 6 are reported (Table 1). The assumed model was a univariate model with a fixed herd effect and a random additive genetic effect. The residual and additive genetic variances were assumed to be 750,000 and $250,000 \mathrm{~kg}^{2}$, respectively. The prior mean vector $(\mathbf{g})$ and the vector of estimated 
Table 1. Design and solutions for example 1

\begin{tabular}{lccccccc}
\hline Animal & Sire & Dam & Herd & $\begin{array}{c}\text { Milk } \\
\text { yield }(\mathrm{kg})\end{array}$ & $\begin{array}{c}\text { Prior } \\
\text { mean }\end{array}$ & Pseudo-record & EBV \\
\hline 1 & - & - & - & - & 0 & -700 & -208.150 \\
2 & - & - & 1 & 8,000 & 200 & 500 & 434.95 \\
3 & 1 & - & 1 & 5,500 & 200 & 1000 & -80.29 \\
4 & 1 & 2 & 2 & 6,000 & 200 & 600 & 122.73 \\
5 & - & 2 & 2 & 6,500 & 0 & -400 & 109.72 \\
6 & - & 3 & 2 & 7,000 & 0 & -400 & 3.62 \\
\hline
\end{tabular}

pseudo-records $\left(\mathbf{y}_{\mathbf{P}}\right)$ are reported in Table 1 . The variance $\mathbf{G}^{-1}$ was equal to $\mathbf{G}^{-1}=\mathbf{A}^{-1}\left(\sigma_{u}^{2}\right)^{-1}$, where $\mathbf{A}^{-1}$ is the inverse of the relationship matrix and $\sigma_{u}^{2}$ is the additive genetic variance. The estimates of the fixed herd effect and the predictions of the random effect were obtained solving the system of equations [4] and [5]. Solved with the free software package GNU Octave (Eaton et al., 2011), both systems of equations provided the same solutions. These results were expected because the 2 systems of equations are equivalent.

\section{Example 2: Integration of External Information Based on a Bayesian Approach}

The example 2 tested the proposed method to integrate external information following the second version of modified Bayesian evaluation (SBE; Vandenplas and Gengler, 2012). The software packages used for this example were ASReml with the !BLUP option (release 3.0; Gilmour et al., 2009), BLUPF90 (version 1.45; Misztal, 2013), and DMU4 from the DMU package (version 6, release 5.1; Madsen and Jensen, 2012).

A local sample of animals and a foreign one, both including about 2,400 animals distributed over 6 generations, were simulated each from 50 male founders and 200 female founders. For both samples, all females were randomly mated with males randomly selected from available males to simulate the next generation. The maximum number of males mated in each generation was 40 and a male could be mated during a maximum of 2 generations. Furthermore, these matings could not be realized if the coefficient of relationship between 2 animals was higher or equal to 0.5, and if the female had already 3 progeny. Concerning the foreign sample, foreign females were only mated with foreign males. In each generation, $60 \%$ of foreign male offspring were randomly culled. Concerning the local sample of animals, local females were mated with local males and a subset of foreign males, including the first 150 sires that had most offspring in the foreign population. In each generation, $99 \%$ of local male offspring were randomly culled. Records for milk yield $(\mathrm{kg})$ for the first lactation were simulated for each female in both samples (Van
Vleck, 1994). A herd effect nested within sample was randomly assigned to each female under the condition that each herd included about 40 females. Residual and additive genetic variances were assumed to be $6 \times 10^{4}$ and $2 \times 10^{4} \mathrm{~kg}^{2}$, respectively.

Simulation of data and a foreign genetic evaluation (i.e., a BLUP evaluation [2] based on foreign pedigree and data) were performed with GNU Octave software. Integration of external information into the local genetic evaluation (i.e., an evaluation based on local pedigree and data) was performed following the SBE. In the context of SBE, external information included foreign EBV for the 150 sires and EBV predicted from the foreign ones for local animals (summarized in $\mathbf{g}$ ) and associated record equivalents only due to own records (RE; i.e., effective numbers of records free of contributions due to relationships estimated by a 2-step algorithm; Vandenplas and Gengler, 2012). Because RE are only due to own records, RE for local animals were equal to zero. The matrix $\mathbf{G}^{-1}$ associated with $\mathbf{g}$ is the matrix of prediction error (co)variances and was approximated as $\mathbf{G}^{-1}=\mathbf{A}^{-1}\left(\sigma_{u}^{2}\right)^{-1}+\boldsymbol{\Lambda}$, where $\boldsymbol{\Lambda}$ is a diagonal matrix with diagonal elements equal to $\boldsymbol{\Lambda}_{i i}=\mathbf{R E}_{i}\left(\sigma_{e}^{2}\right)^{-1}$ for the $i$ th animal (Vandenplas and Gengler, 2012). The SBE was also performed with GNU Octave software and obtained solutions were considered as reference.

To test the proposed approach with ASReml, BLUPF90, and DMU4, local pedigree and data files as well as foreign EBV for the 150 sires and predicted EBV for local animals and associated RE were created. Setting up of $\mathbf{G}^{-1}, \mathbf{g}, \mathbf{Z}_{\mathbf{P}}$, and $\mathbf{R}_{\mathbf{P}}$, and computations of $\boldsymbol{\theta}, \mathbf{y}_{\mathbf{P}}$, and $\mathbf{G}^{*-1}$ were performed with GNU Octave software. Files containing the user-supplied (co)variance matrix were written following specific rules for each software package and the pseudo-records in $\mathbf{y}_{\mathbf{P}}$ were added to the data file with appropriate levels for the different effects.

The system of equations [5], based on the extended data and user-supplied (co)variance matrix, was solved with ASReml, BLUPF90, and DMU4. For DMU4, solutions were obtained with the equivalent nested regres- 
sion model. The system of equations was solved by direct computation proposed by the 3 software packages used. Solutions were compared with those estimated with GNU Octave software, measuring the relative errors

$$
\Delta=\left|\frac{s o l_{i j}-s o l_{O j}}{s o l_{O j}}\right|,
$$

where $\operatorname{sol}_{O j}$ was the estimate for the $j$ th level of the effects estimated with GNU Octave and $s o l_{i j}$ was the estimate for the $j$ th level of the effects estimated with 1 of the 3 software packages used (i.e., $i=$ ASReml, BLUPF90, or DMU4). The $\Delta$ values ranged from 0.00 to $4.96 \times 10^{-6}$ for ASReml, from 0.00 to $9.78 \times 10^{-8}$ for BLUPF90, and from 0.00 to $3.83 \times 10^{-4}$ for DMU4. Average $\Delta$ were $9.11 \times 10^{-7}$ for ASReml, $2.54 \times 10^{-10}$ for BLUPF90, and $2.52 \times 10^{-6}$ for DMU4. Differences between $\Delta$ can be explained by the precision considered by the software packages for integer and real variables and by the writing format specifications of the software packages for the solutions.

In conclusion, these 2 examples showed the possibility to alter both mean and (co)variance of the prior distribution associated with random effects for linear mixed models equations with current software packages commonly available in the animal breeding community through the use of an extended data file and a usersupplied (co)variance matrix.

\section{ACKNOWLEDGMENTS}

J. Vandenplas, as a research fellow, and N. Gengler, as a former senior research associate, acknowledge the support of the National Fund for Scientific Research (Brussels, Belgium) for these positions. Additional financial support was provided by the Ministry of Agriculture of Walloon Region of Belgium (Service Public de Wallonie, Direction générale opérationnelle "Agriculture, Ressources naturelles et Environnement" - DGARNE) through research projects D31-1207,
D31-1224, and D31-1274. Useful comments from the 2 reviewers are also acknowledged.

\section{REFERENCES}

Dempfle, L. 1977. Relation entre BLUP (best linear unbiased prediction) et estimateurs bayésiens. Ann. Genet. Sel. Evol. 9:27-32.

Eaton, J. W., D. Bateman, S. Hauberg, and R. Wehbring. 2011. GNU Octave. A high-level interactive language for numerical computations. 3rd ed. Free Software Foundation Inc., Boston, MA.

Gianola, D., and R. L. Fernando. 1986. Bayesian methods in animal breeding theory. J. Anim. Sci. 63:217-244.

Gilmour, A. R., B. J. Gogel, B. R. Cullis, and R. Thompson. 2009. ASReml User Guide. Release 3.0. VSN International Ltd., Hemel Hempstead, UK.

Henderson, C. R. 1950. Estimation of genetic parameters. Ann. Math. Stat. 21:309. (Abstr.)

Henderson, C. R. 1975. Best linear unbiased estimation and prediction under a selection model. Biometrics 31:423-447.

Legarra, A., J. K. Bertrand, T. Strabel, R. L. Sapp, J. P. Sanchez, and I. Misztal. 2007. Multi-breed genetic evaluation in a Gelbvieh population. J. Anim. Breed. Genet. 124:286-295.

Lindley, D. V., and A. F. M. Smith. 1972. Bayes estimates for the linear model. J. R. Stat. Soc. Ser. B Methodol. 34:1-41.

Madsen, P., and J. Jensen. 2012. A user's guide to DMU. A package for analysing multivariate mixed models. Version 6 , release 5.1. University of Aarhus, Faculty of Agricultural Sciences (DJF), Dept. of Genetics and Biotechnology, Research Centre Foulum, Tjele, Denmark.

Misztal, I. 2013. BLUPF90 family of programs. Accessed July 3, 2012. http://nce.ads.uga.edu/wiki/doku.php.

Quaas, R. L. 1984. Linear prediction. Pages 1-76 in BLUP School Handbook. Use of Mixed Models for Prediction and for Estimation of (Co)variance Components. Animal Genetics and Breeding Unit, University of New England, New South Wales, Australia.

Quaas, R. L., and Z. Zhang. 2006. Multiple-breed genetic evaluation in the US beef cattle context: Methodology. CD-ROM commun. 24-12 in Proc. 8th World Congr. Appl. Livest. Prod., Belo Horizonte, Brazil.

Schaeffer, L. R., and J. Jamrozik. 1996. Multiple-trait prediction of lactation yields for dairy cows. J. Dairy Sci. 79:2044-2055.

Sorensen, D., and D. Gianola. 2002. Likelihood, Bayesian and MCMC Methods in Quantitative Genetics. Springer-Verlag, New York, NY.

Van Vleck, L. D. 1994. Algorithms for simulation of animal models with multiple traits and with maternal and non-additive genetic effects. Rev. Brasil. Genet. 17:53-57.

Vandenplas, J., and N. Gengler. 2012. Comparison and improvements of different Bayesian procedures to integrate external information into genetic evaluations. J. Dairy Sci. 95:1513-1526.

Vitezica, Z. G., I. Aguilar, I. Misztal, and A. Legarra. 2011. Bias in genomic predictions for populations under selection. Genet. Res. (Camb.) 93:357-366. 